\title{
Culture Moderates Biases in Search Decisions
}

Jake A. Pattaratanakun and Vincent Mak

March 31, 2015

Forthcoming in Psychological Science

\section{Author Note}

Jake A. Pattaratanakun, Cambridge Judge Business School, University of Cambridge; Chulalongkorn Business School, Chulalongkorn University

Vincent Mak, Cambridge Judge Business School, University of Cambridge

Correspondence concerning this article should be addressed to Vincent Mak, Cambridge Judge Business School, University of Cambridge, Trumpington Street, Cambridge CB2 1AG, United Kingdom, Phone: +44(0)1223-764295, Fax: +44(0)1223-339701, Email:

v.mak@jbs.cam.ac.uk 


\title{
Culture Moderates Biases in Search Decisions
}

\begin{abstract}
Prior studies suggest that people often searched insufficiently in sequential search tasks compared with benchmark optimal strategies that maximize expected payoff. We point out that those studies were mostly conducted in individualist Western cultures; Easterners from collectivist cultures, with their higher susceptibility to escalation of commitment induced by sunk search costs, could exhibit a reversal of this under-search bias by searching more than optimally (only) when search costs are high. We tested our theory in four experiments. In our pilot experiment, participants generally under-searched when search cost was low, but only Eastern participants over-searched when search cost was high. Experiments 1 and 2 offered evidence for our hypothesized effects via a cultural priming manipulation on bicultural participants through interface language. We obtained further process evidence for our theory in Experiment 3, in which we made sunk costs non-salient in the search task - as expected, crosscultural effects became largely mitigated.
\end{abstract}

Keywords: search, culture, individualism vs. collectivism, sunk costs, escalation of commitment, biculturalism, priming by interface language 


\section{Culture Moderates Biases in Search Decisions}

Search is a very general class of sequential decision making. In essence, it involves trading off between the opportunity to acquire more information or options that can benefit decision, and the cost of such an acquisition. As an illustration, consider a scenario in which a new model of a popular smartphone is out in the market. A consumer may visit one retailer after another, examining their offers one at a time, before making a purchase. At each visit, the consumer has to decide whether to stop at that point to buy the best available offer encountered so far, or to visit yet another retailer, which will incur a search cost (in terms of time, effort, transportation, etc.) but potentially lead to the discovery of a better deal.

Theories of rational decisions have provided "optimal stopping" strategies that maximize expected payoff in models of search (DeGroot, 1970; Weitzman, 1979). Prior experimental research on these models found that people often searched insufficiently compared with optimal benchmarks (e.g., Seale \& Rapoport, 1997, and the review in Bearden \& Rapoport, 2005). Such "under-search" bias is consistent with empirical findings that consumers engaged in rather limited search for a wide range of products (Beales, Mazis, Salop, \& Staelin, 1981; Dickson \& Sawyer, 1990; Moorthy, Ratchford, \& Talukdar, 1997), and anecdotal evidence that grocery consumers searched less than retail executives expected (see e.g., Urbany, Dickson, \& Kalapurakal, 1996). But Zwick, Rapoport, Lo, and Muthukrishnan (2003) provided a contravening exception to these results: in their experiment, participants tended to search too much ("over-search") relative to the optimal stopping strategy (only) when search costs were high. It has never been completely clarified how such deviations from previous findings could have occurred. 
The present research explores the explanation that Zwick et al. (2003)'s experiments were conducted with culturally collectivist (Hofstede, 1980; Triandis, 1995) Eastern participants in Hong Kong, whereas previous studies were largely conducted with participants from individualist Western cultures. That is, the individualism-collectivism dimension of culture might have a moderating effect on participants' biases in search decisions relative to optimal stopping strategies. However, Zwick et al. (2003) suggest that the posited effects appear only at high search costs; that is, search decisions are not straightforwardly impacted by culture, but by an interaction of cross-cultural differences (along the individualism-collectivism dimension) and search costs. This motivates the following conceptual development for our study.

\section{Conceptual Development: Search, Escalation of Commitment, and Culture}

Standard economic theorizing prescribes that, over the process of a sequential search task, decision makers (DMs) should only evaluate the costs and expected gains of additional searches. However, prior research (e.g., Kogut, 1990) suggests that DMs would often be influenced by sunk costs of previous searches. It can also be expected that sunk costs would influence DMs via escalation of commitment, i.e., strengthening the commitment to continue to search, instead of choosing to stop searching, over and above standard economic concerns (Thaler, 1980; Arkes \& Blumer, 1985). Previous studies suggest that risk seeking people are especially susceptible to this effect (Philips, Battalio, \& Kogut, 1991; Keil et al., 2000). Lastly, it has been observed that Easterners (Westerners) appeared to be risk seeking (risk averse) in financial decisions (Weber \& Hsee, 1998; Hsee \& Weber, 1999). This has been interpreted as resulting from the contrast in collectivism versus individualism between Eastern and Western cultures. As Weber \& Hsee (1998, p.1208) explained, in collectivist cultures, "family or other ingroup members will step in to help out any group member who encounters a large and possibly 
catastrophic loss after selecting a risky option. In individualist cultures ... a person making a risky decision will be expected to personally bear the (possibly adverse) consequences of their decisions. Collectivism thus acts as a cushion against possible losses."

To sum up, there appears to be a qualitative difference in risk attitude between Easterners and Westerns, which is driven by the collectivism/individualism contrast. This difference could then lead to a difference in susceptibility to sunk cost-induced escalation of commitment. Consistently, Keil et al. (2000) found that Easterners had a higher inclination toward escalation of commitment than Westerners in the domain of software project development, largely because of more risk taking. Applying this insight to search decisions, we conjecture that, because of Easterners' higher susceptibility to sunk cost-induced escalation of commitment, Easterners are more inclined towards over-search than Westerners are when sunk search costs become high.

\section{Overview of the Experimental Setup}

To examine our conjecture, we carried out four experiments using a simple price search task that is based on a paradigm with a long usage (see e.g., Rapoport \& Tversky, 1970). In our setup, a DM has to purchase one unit of a virtual product with a known value, and obtains price quotes for the product one at a time. Every time the DM obtains a price quote, he/she makes a choice between stopping search to make a purchase, and committing to continue to search in the sense of obtaining one more price quote at a known search cost. Price quotes are independently and identically distributed random variables with a distribution function known by the DM before the search begins. All searched offers are always available (perfect recall), so that a DM who has stopped is assumed to purchase at the minimum price quote he/she has encountered. The DM's payoff from a purchase is thus the value of the product minus the sum of the minimum price quote obtained and the total search cost incurred. 
In our pilot experiment, search costs were constant with search; we compared the decisions of Western/Eastern participants from distinctly individualist/collectivist cultures at different search costs, in order to obtain support for our basic theoretical premises. In Experiments 1 to 3, search costs increased with search. Experiments 1 and 2 were designed to offer evidence for our hypothesized effects via a cultural priming manipulation on bicultural participants through interface language. In these two experiments, as in the pilot experiment, the participant was provided with the total incurred search cost (and by implication, the sunk cost) at all points of decision. In Experiment 3, we omitted such information at all points of decision to make sunk costs non-salient; our aim was to examine if cross-cultural differences in search decisions would be mitigated, as our theory predicts.

\section{Pilot Experiment}

\section{Method}

Participants. One hundred and eight students from a UK (32 females, 13 males) and a Thai (44 females, 19 males) university participated in the experiment. Both universities were ranked among the top in their countries; the experiment had received ethical approval from the Psychology Research Ethics Committee of the UK participants' university, as well as from the Thai participants' university. The participants were recruited from a pool of behavioral experiment subjects in their university over a one-month period up to the date of the experimental session. The participants were made aware that they would be participating, with payment, in a decision making study that would last approximately one hour. Only participants who were national citizens were allowed to participate, to ensure consistency in cultural background. The country comparison data at the Hofstede Centre website (Hofstede Centre, 2014) show that both cultures score moderately low to moderately high on five of the six cultural 
dimensions listed: respectively for UK vs. Thailand, the scores are (out of 100) 35 vs. 64 on power distance, 66 vs. 34 on masculinity, 35 vs. 64 on uncertainty avoidance, 51 vs. 32 on long term orientation, and 69 vs. 45 on indulgence. However, the two cultures are very different on the remaining dimension, namely the individualism score: 89 in the UK versus 20 in Thailand.

Design and procedure. The experiment had a 2 (culture: UK vs. Thai) $\times 2$ (search cost: low vs. high) between-subjects design. Across all conditions, every price quote was generated by a random draw (rounded to the nearest integer) from a normal distribution with mean $=430$ tokens and standard deviation $=50$ tokens, token being the experimental currency used throughout our research. The value of the virtual object of search was controlled to be 700 tokens in all conditions. The participants' earnings in the experimental currency were converted to real currency for payment at the end of the experiment.

Every participant played 45 search games with the same search cost. These included five practice games, followed by 40 actual games for payment. In each game, the participant visited one or more "shops" to purchase one product item with the value of 700 tokens. With each visit, the participant obtained a price quote that was randomly generated according to the normal distribution defined earlier. The participant visited the first shop with no cost, but afterwards every additional visit incurred a constant search cost. This search cost was manipulated at two levels across conditions: 5 tokens per search (low search cost) and 15 tokens per search (high search cost). The participant was provided with the total incurred search cost (i.e., the sunk cost) at all points of decision. Upon making a purchase, the participant paid a purchase price that was equal to the minimum price quote obtained during the search. Each participant could view a history screen at any time to access the price quotes obtained so far in the search. The 
participant's net payoff in the game was the product value of 700 tokens minus the search costs incurred over the course of the game, and the purchase price.

A sample of the experimental instructions, including sample decision and feedback screens of the experimental program interface, is included in Appendix B. The program was written using the z-Tree software (Fischbacher, 2007). All participants conducted the task with instructions and program interface in their native language. Two professional translators made double back translations of all textual materials from English to Thai and from Thai to English to ensure consistency across conditions. We also ensured that the participants understood the search task by having them complete a quiz after reading the instructions (see Appendix B). As with the other experiments in this research, almost all participants completed their quiz correctly, and any erroneous answers were pointed out to the participant by the experimenter with a full explanation before the experiment began.

Three games were randomly selected after the experiment was over. Each participant's payment was his/her average earnings from those three games converted to real currency at the rate of 1 token $=£ 0.01$ for the UK sessions $(£ 1 \approx$ US\$1.6) and 1 token $=0.13$ Thai baht for the Thai sessions ( 1 Thai baht $\approx$ US\$0.033). These exchange rates were designed so that one token was worth about three to four times in US dollars to a UK participant compared with a Thai participant; this was approximately the ratio of the two countries' gross domestic product per capita based on purchase-power-parity in current international dollars over 2010 to 2013 (International Monetary Fund, 2013), when our experiments were conducted. In addition, each participant was paid a show-up fee of $£ 3$ in the UK sessions and 40 Thai baht in the Thai 
UK, Low search cost

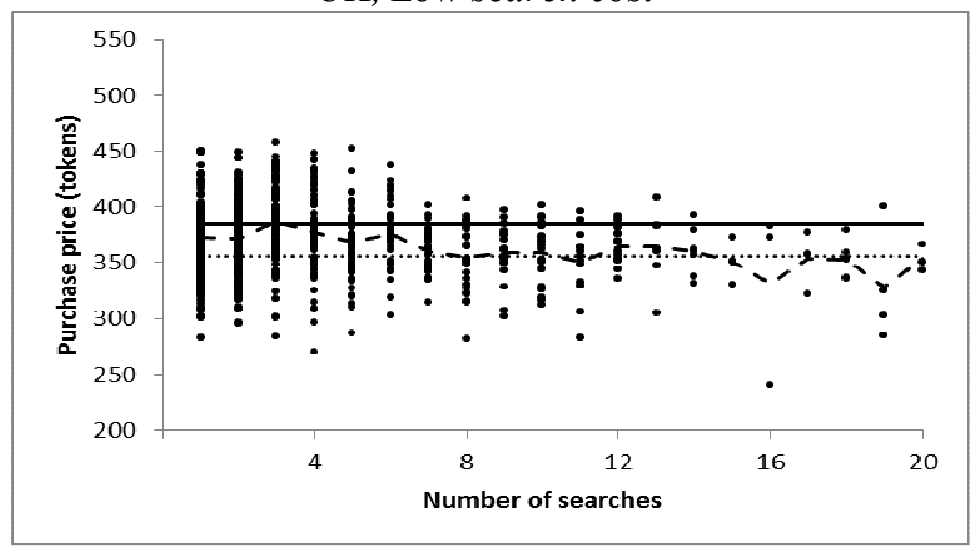

UK, High search cost

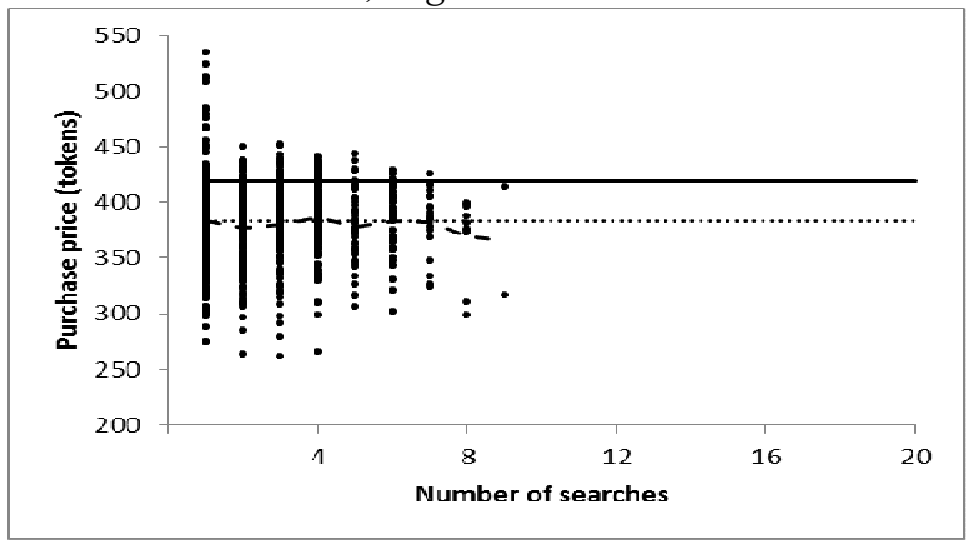

Thai, Low search cost

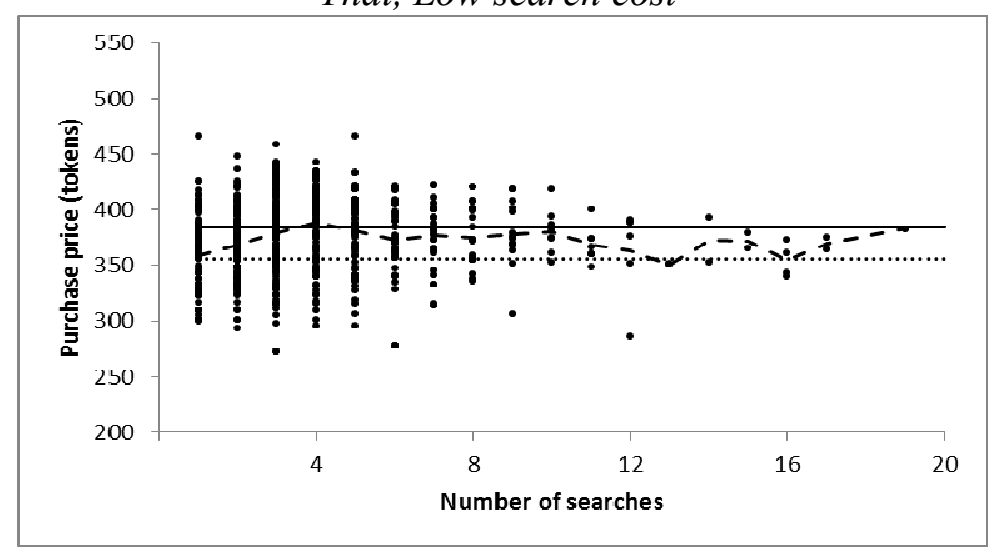

Thai, High search cost

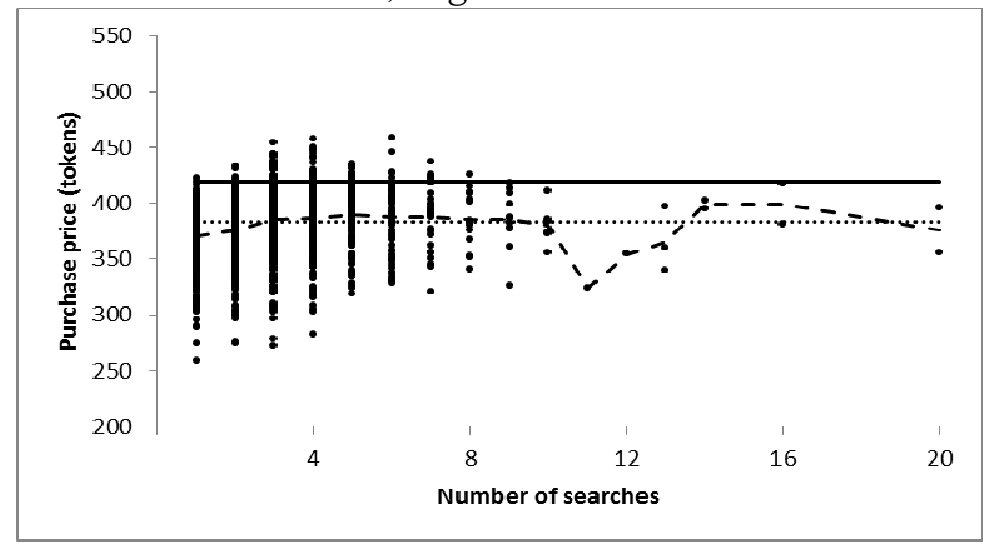

Fig. 1. Observed purchases in the pilot experiment. Each dot represents one observed purchase. The dashed lines mark the mean observed purchase price by number of searches. The dotted lines mark the theoretical expected purchase prices under the optimal strategy. The straight lines mark the threshold prices for acceptance under the optimal strategy; that is, under the optimal strategy, no purchase observations should lie above the straight lines. 
sessions. Average participant payment was $£ 6$ in the UK sessions and 78.5 Thai baht in the Thai sessions.

\section{Results}

In the low search cost conditions, an expected payoff-maximizing DM should stop searching as soon as he obtained a price quote that was not higher than 384 tokens (see Appendix A). In the high search cost conditions, the corresponding threshold was 419 tokens. These thresholds form the benchmark optimal strategies against which we compare participants' decisions. The panels in Fig. 1 present the observed purchases in the experiment in each condition alongside benchmark predictions. In the figure, each dot represents a purchase characterized by the number of searches and the purchase price. The dashed lines mark the mean observed purchase price by number of searches. The dotted lines mark the theoretical expected purchase prices under the optimal strategy. Lastly, the straight lines mark the threshold prices for acceptance under the optimal strategy; dots above the straight lines are symptomatic of undersearching and over-paying with respect to the optimal benchmarks.

Comparing the panels across cultures controlling for the level of search cost, we observe that: (a) the patterns of purchase observations at low search cost are largely the same across cultures and indicate a tendency for under-search biases; but (b) there are marked cross-cultural differences at high search cost, characterized by more long searches with low purchase prices among the Eastern participants compared with the Western participants. These observations are consistent with our general premise that Easterners search more than Westerners only when search cost is high, when sunk cost has high impact by implication.

Table 1 lists the main results from the experiment, and indicates whether an observed mean in a condition is significantly different from the benchmark expected value in the 
"Optimal" columns. The table thus suggests that, consistent with our theorizing: (a) both cultures under-searched and over-paid at low search cost with respect to the optimal benchmarks; (b) the Western participants searched optimally at high search cost; (c) the Eastern participants oversearched and under-paid at high search cost.

Table 1. Main Results from the Pilot Experiment

\begin{tabular}{|c|c|c|c|}
\hline & \multicolumn{3}{|c|}{ Mean number of searches } \\
\hline & UK & Thai & Optimal \\
\hline Low search cost & $\begin{array}{c}4.17 \begin{array}{c}(1.65)[3.44,4.90] \\
p=.001 ; N=22\end{array}\end{array}$ & $\begin{array}{c}3.79(1.01)[3.35,4.22] \\
p<.001 ; N=23\end{array}$ & 5.48 \\
\hline \multirow[t]{3}{*}{ High search cost } & $\begin{array}{c}\frac{2.55}{p}(0.57)[2.30,2.79] \\
p>.250 ; N=23\end{array}$ & $\begin{array}{c}\frac{2.83}{(0.37)}[2.71,2.95] \\
p<.001 ; N=40\end{array}$ & 2.42 \\
\hline & \multicolumn{3}{|c|}{ Mean purchase price } \\
\hline & UK & Thai & Optimal \\
\hline Low search cost & $\begin{array}{c}372(15.54)[365,379] \\
p<.001\end{array}$ & $\begin{array}{c}375(9.06)[371,379] \\
p<.001\end{array}$ & 358 \\
\hline High search cost & $\begin{array}{c}381(12.09)[376,386] \\
p>.250\end{array}$ & $\begin{array}{c}380(5.11)[378,381] \\
p<.001\end{array}$ & 383 \\
\hline
\end{tabular}

Note: The entries were calculated with participant as the unit of observation; the values in parentheses are the SDs and those in square brackets are $95 \%$ confidence intervals. The underlined entries highlight a significant effect of culture according to $t$-test $(p=.019)$ that is discussed in more detail in the Results section for this experiment. The column "Optimal" lists the expected value of the relevant dependent variable in the experiment under the expected payoff-maximizing strategy. The $p$ values indicate the results from comparing the mean of the corresponding dependent variable with its expected value under the optimal strategy by $t$-test. The experimental task instructions and interfaces were in the participant's native language in every condition.

Table 1 suggests an interaction effect with the number of searches. Indeed, a 2 (culture: UK vs. Thai) $\times 2$ (search cost: low vs. high) between-subjects ANOVA for the number of searches does not yield a significant effect of culture $\left(F(1,104)=0.06, p>.250, \eta_{p}^{2}<.001\right)$, but 
reveals a marginally significant interaction $\operatorname{effect}\left(F(1,104)=3.24, p=.075, \eta_{p}^{2}=.030\right)$. We follow up with $t$-tests for simple effects of culture at each level of search cost; we do not find a significant effect at low search cost $(t(43)=0.95, p>.250$, Cohen's $d=0.28)$ but find a significant effect at high search cost $(t(61)=2.42, p=.019$, Cohen's $d=0.62)$. That is, our Eastern participants made a similar number of (more) searches compared with Western participants at low (high) search cost. Nevertheless, the two cultures did not purchase at very different prices on average controlling for search cost: a similar ANOVA with purchase price as the dependent variable does not yield a significant main effect of culture nor any significant interaction in both cases (main effect of culture: $F(1,104)=0.18, p>.250, \eta_{p}^{2}=.002$; interaction: $\left.F(1,104)=1.29, p>.250, \eta_{p}^{2}=.012\right)$.

\section{Discussion}

The pilot experiment lends some support to our theoretical premises: at low search cost, both Western and Eastern participants - from distinctly individualist and collectivist cultures under-searched and purchased at higher prices compared with the optimal benchmarks; but at high search cost, collectivist Eastern participants over-searched and under-paid with respect to the optimal benchmarks, while individualist Western participants searched optimally.

\section{Experiment 1}

While the pilot experiment established preliminary evidence for our premise, a serious concern is that the participant populations representing different cultures might differ in many non-culture-specific aspects that might affect their search decisions. Experiment 1 was designed to remedy the concern by culturally priming participants from a bicultural population via the task interface language. 
Bicultural participants exhibit mental "frame switching" when primed with one or the other of their cultures (see e.g., Gardner, Gabriel, \& Lee, 1999; Hong, Morris, Chiu, \& BenetMartinez, 2000; Sui \& Han, 2007). Previous studies show that language can be an effective means to achieve this form of priming (e.g., Luna, Ringberg, \& Peracchio, 2008; Ross, Xun, \& Wilson, 2002). Of particular relevance is the research by Kemmelmeier and Cheng (2004), who demonstrated that, among their bicultural participants, a Western (Eastern) language could activate self-construals that were normative in individualist (collectivist) cultures (see also Hoffman, Lau, \& Johnson, 1986, for a related study). Accordingly, we posit that, in our search framework, interface language could be used as a cultural priming manipulation for a bicultural population. That is, bicultural participants' decision biases would be moderated by the interface language, so that they are more prone to over-search bias when performing the experimental task in their Eastern language than in their Western language.

\section{Method}

Participants. One hundred twenty participants, who did not take part in the pilot experiment, participated in Experiment 1. The UK (23 females, 13 males) and Thai (53 females, 31 males) participants were students from the same universities as in the pilot experiment; the experiment had also received ethical approval from the same authorities as in the pilot experiment. The participants were recruited via similar procedures as in the pilot experiment, except that the Thai participants were Thai nationals recruited from an international business program in which English was the official language of instructions. Those participants had been typically brought up in a bicultural, bilingual atmosphere, such as an international school system. They had been habitually exposed to Western culture through their course materials (almost all of them having made at least one semester of exchange visit to the West), as well as interactions 
with hundreds of Western exchange students and Western educated faculty. Moreover, the students generally accessed both Thai and English-language entertainment and news on a regular basis. To sum up, the Thai participants in this experiment were from a highly westernized Eastern population who were bicultural to a comparable extent as bicultural participants in previous cross-cultural studies (e.g., Hong et al., 2000; Kemmelmeier \& Cheng, 2004). The participants were made aware that they would be participating, with payment, in a decision making study that would last approximately one hour.

Design and procedure. Experiment 1 had a 3(UK vs. Thai (English interface) vs. Thai (Thai interface)) between-subjects design. All conditions involved the same search task, which was identical to that in the pilot experiment except that search cost increased proportionally with search. Specifically, the cost of obtaining one more price quote after having obtained $i$ price quotes was $5 i$. Implementing a search task with increasing search cost added more interest to our experiment, as it went beyond the constant search cost paradigm in the pilot experiment that is typical of much of relevant previous research. Moreover, it reflects realistic situations where search cost increases with search (e.g., a consumer might begin search around familiar shops in the neighborhood, but further searches would involve travelling to less familiar shops in more distant locations). Lastly, in this setting, the total sunk search cost - which was provided to the participant at all points of decision (cf. the left panel of Fig. 4) - increased rapidly with searches. This could make cross-cultural differences in susceptibility to escalation of commitment more pronounced than the high search cost condition in the pilot experiment, but without the need to impose a prohibitively high search cost from the first search onward.

Average participant payment was $£ 5.9$ in the UK sessions, 77 Thai baht in the Thai (English interface) sessions, and 75.4 Thai baht in the Thai (Thai interface) sessions. 


\section{Results}

In the experiment, an expected payoff-maximizing DM should search with a threshold price that increased as the number of searches increased (see Appendix A). This forms the benchmark optimal strategy against which we compare participants' decisions. The panels in Fig. 2 present the observed purchases in the experiment in each condition alongside benchmark predictions, with similar notations as in Fig. 1. Comparing the figures across conditions suggests that, as expected: (a) the patterns of purchase observations are largely the same between the Western participants and the bicultural participants conducting the experiment in English; but (b) these two patterns are markedly different from that of the bicultural participants conducting the experiment in their Eastern language. The main difference is that the latter tended to make more long searches before making purchases.

Table 2 lists the main results from the experiment, and indicates whether an observed mean in a condition is significantly different from the benchmark expected value in the "Optimal" columns. The table suggests that: (a) the Western participants searched approximately optimally; (b) the bicultural participants, when conducting the experiment in their Eastern language, tended to over-search and under-pay with respect to the optimal benchmarks. We carry out $t$-tests comparing the Western participants with the bicultural participants conducting the experiment in their Eastern language, with number of searches and purchase price as the dependent variables. Both tests yield significant effects (number of searches: $t(83)=-3.68, p<$ .001 , Cohen's $d=0.81$; purchase price: $t(83)=4.17, p<.001$, Cohen's $d=0.92$ ). It appears that, with the more rapidly increasing sunk search cost in this experiment compared with the pilot experiment, cross-cultural differences were more prominent than in the earlier experiment. 


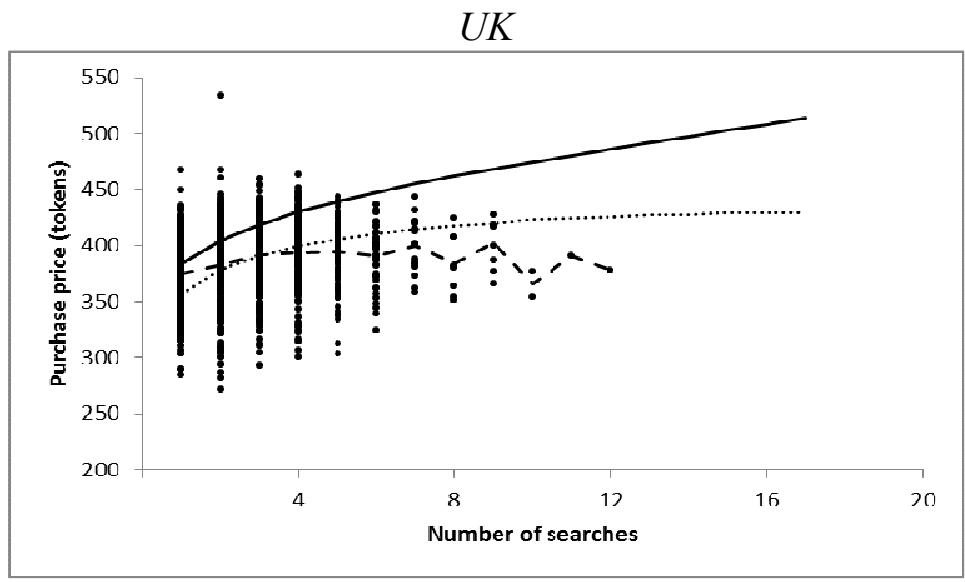

Thai (English interface)

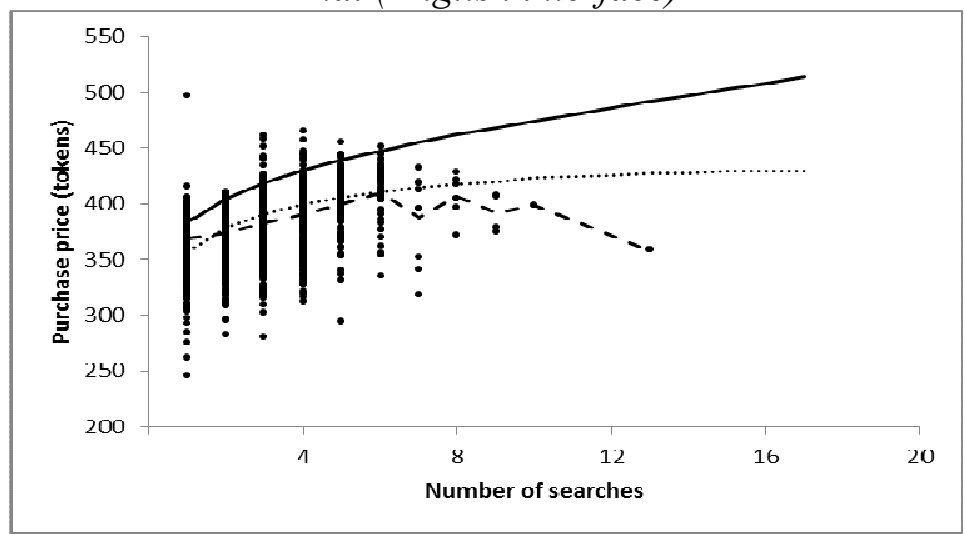

Thai (Thai interface)

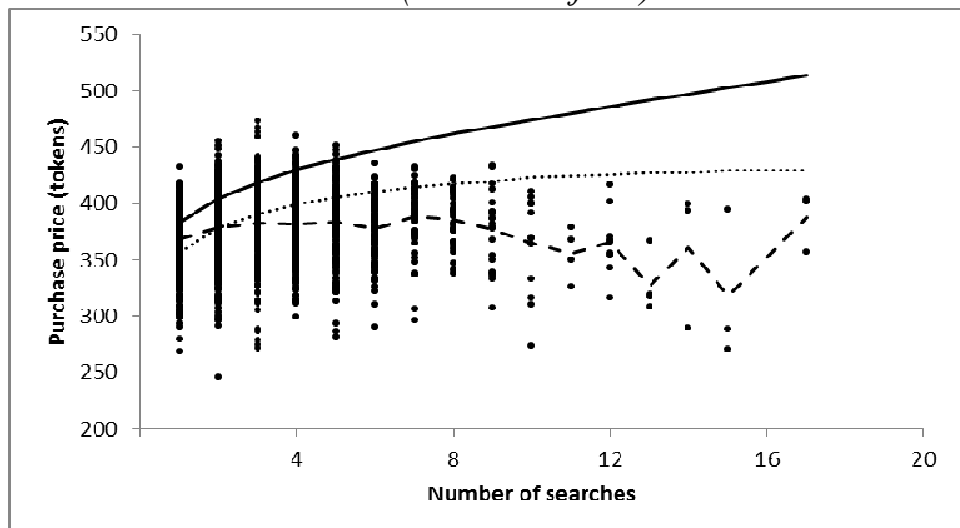

Fig. 2. Observed purchases in Experiment 1. See the caption under Fig. 1 for notations. 
Table 2. Main Results from Experiment 1

\begin{tabular}{|c|c|c|c|}
\hline \multicolumn{4}{|c|}{ Mean number of searches } \\
\hline UK & $\begin{array}{c}\text { Thai } \\
\text { (English interface) }\end{array}$ & $\begin{array}{c}\text { Thai } \\
\text { (Thai interface) }\end{array}$ & Optimal \\
\hline $\begin{array}{c}2.69(0.50)[2.52,2.86] \\
p=.206 ; N=36\end{array}$ & $\begin{array}{c}2.84(0.31)[2.73,2.95] \\
p<.001 ; N=35\end{array}$ & $\begin{array}{c}3.23(0.77)[3.01,3.45] \\
p<.001 ; N=49\end{array}$ & 2.58 \\
\hline \multicolumn{4}{|c|}{ Mean purchase price } \\
\hline UK & $\begin{array}{c}\text { Thai } \\
\text { (English interface) }\end{array}$ & $\begin{array}{c}\text { Thai } \\
\text { (Thai interface) }\end{array}$ & Optimal \\
\hline $\begin{array}{c}385(9.13)[382,388] \\
p=.147\end{array}$ & $\begin{array}{c}381(5.19)[380,383] \\
p=.076\end{array}$ & $\begin{array}{c}378(6.60)[376,380] \\
p<.001\end{array}$ & 383 \\
\hline
\end{tabular}

Note: See the note under Table 1 for notations, except that every bold entry highlights a significant difference from the comparable entry in the Thai (Thai interface) condition according to $t$-test $(p<.05)$, which is discussed in more detail in the Results section for this experiment.

Table 2 also suggests that: (a) while the bicultural participants in the English language condition indeed over-searched, the extent was notably less than that of the other bicultural participants who conducted the experiment in their Eastern language; (b) the bicultural participants in the English language condition only marginally under-paid with respect to the optimal benchmark. Both observations are consistent with our hypothesized moderating effect of culture. Pairwise $t$-test comparisons show that there are significant differences between the two groups of bicultural participants conducting the experiment in their Western versus Eastern languages (number of searches: $t(82)=-2.84, p=.006$, Cohen's $d=0.63$; purchase price: $t(82)=2.42, p=.018$, Cohen's $d=0.53)$. The differences are both in directions that bring the bicultural participants conducting the experiment in English closer to the Western participants compared with the other bicultural participants.

\section{Discussion}


Experiment 1 lends further support to our theorizing by: (a) demonstrating similar effects as found in the pilot experiment for a search task with increasing search cost, and (b) showing that such differences persisted or are largely mitigated depending on the experimental interface language used by the bicultural participants.

\section{Experiment 2}

Experiment 2 aims to strengthen our evidence from Experiment 1 by means of a withinsubjects design. We also obtained supporting process evidence to our theorizing by comparing measures of participants' susceptibility to sunk cost-induced escalation of commitment at the end of every session.

\section{Method}

Participants. Sixty-two bicultural Thai (48 females, 14 males) participants, who were recruited from the same international business program as the participants in Experiment 1 via similar procedures, took part in both sessions of the experiment (two participants took part in the first session but did not attend the second one; their data are not included in the analysis reported here). The participants were from a different cohort than those in Experiment 1, and did not take part in the pilot experiment either. The experiment had received ethical approval from the participants' university. The participants were made aware that they would be participating, with payment, in a decision making study that would last approximately one hour.

Design and procedure. The experiment consisted of two sessions separated by approximately three months. The setup of every session, including the search task and the manipulation via interface language (as well as the provision of the total sunk search cost to the participant at all points of decision), was identical to that in Experiment 1. In the first session, the participants conducted the experiment in either English (30 participants) or Thai (32 
participants). In the second session, a participant conducted the experiment in Thai if he/she conducted the first session in English, and vice versa. As result, every participant conducted the experiment in his/her Eastern language in one session and his/her Western language in the other session, while the order of interface language across sessions was counterbalanced among the participants. Average participant payment was 77.3 Thai baht in the first session and 79 Thai baht in the second session.

Process measures. At the end of each session, we surveyed every participant for his/her susceptibility to sunk cost-induced escalation of commitment using a three-question questionnaire. The questions were adapted from the scenarios in Experiments 1 and 6 in Arkes and Blumer (1985) and Example 5 in Thaler (1980). The questionnaire was presented in the same language as the interface language with which the participant conducted the experiment in the session just completed (see Appendix $\mathrm{C}$ for the English version of the questionnaire). Results

We first check for order effect by examining the interaction effect in a 2 (order of interface language across sessions: English then Thai vs. Thai then English) $\times 2$ (interface language: English vs. Thai) mixed-design ANOVA for the number of searches, where the first factor is between-subjects while the second factor is within-subjects. The analysis yields a marginally significant interaction effect $\left(F(1,60)=3.15, p=.081, \eta_{p}^{2}=.050\right)$. A similar mixeddesign ANOVA for the purchase price does not yield a significant interaction effect $\left(F(1,60)=1.52, p=.22, \eta_{p}^{2}=.025\right)$. We conclude that the order of interface languages had not exerted an overall significant influence on our major results - which focus on within-subjects comparisons between search decisions under different interface languages. Henceforth we shall analyze the data as in a simple two-factor (interface language: English vs. Thai) within-subjects design. 
The panels in Fig. 3 present the observed purchases in each interface language condition with similar notations as in Fig. 1. The patterns of purchase observations are consistent with those for the bicultural participants in Experiment 1. That is, participants tended to make more long searches before making purchases when conducting the experiment in their Eastern (compared with Western) language.

Table 3 lists the main results from the experiment, and indicates whether an observed mean in a condition is significantly different from the benchmark expected value. The table suggests findings that are similar to those in Experiment 1. That is, the participants over-searched and under-paid with respect to the optimal benchmarks in both conditions, but their biases were notably more severe when they conducted the experiment in their Eastern (compared with Western) language. In other words, the same bicultural participants under different cultural priming manipulations had their search decisions moderated in our hypothesized directions. Our observations are further supported by within-subjects ANOVA for the two major dependent variables, which reveal significant differences in the hypothesized directions (number of searches: $F(1,61)=54.21, p<.001, \eta_{p}^{2}=.47$; purchase price: : $F(1,61)=54.21, p=.001$, $\left.\eta_{p}^{2}=.16\right)$

Process evidence. Table 4 lists the main results from the process measure questionnaire administered at the end of each session. In our analysis, participants' replies to each question are converted into scores such that a higher score indicates a higher susceptibility to sunk costinduced escalation of commitment (cf. the coding explanation in the first column of Table 4). As mentioned before, each participant completed the questionnaire in the same language as the interface language by which he/she conducted the experiment in the session just completed; the results in Table 4 are thus listed separately for the different languages. 


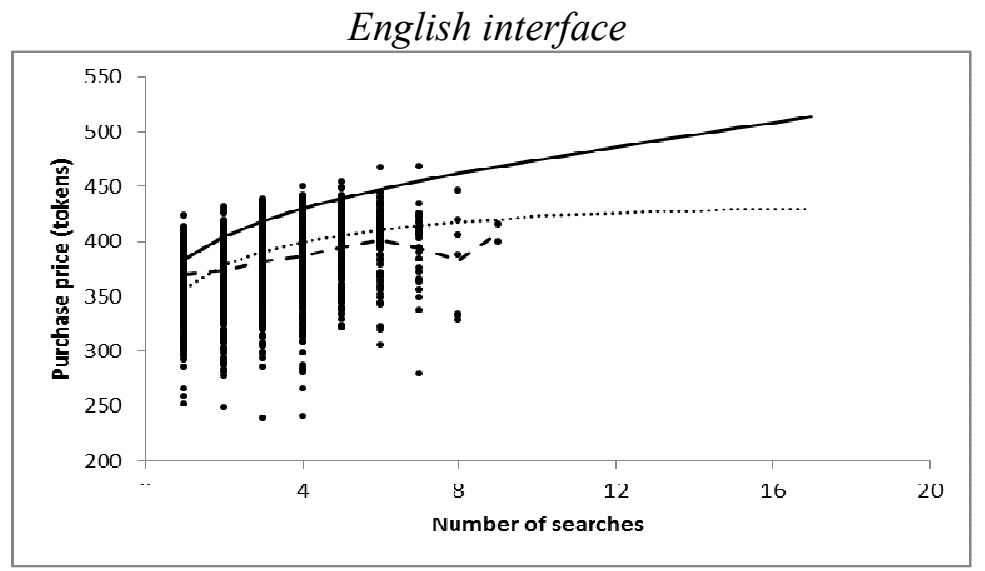

Thai interface

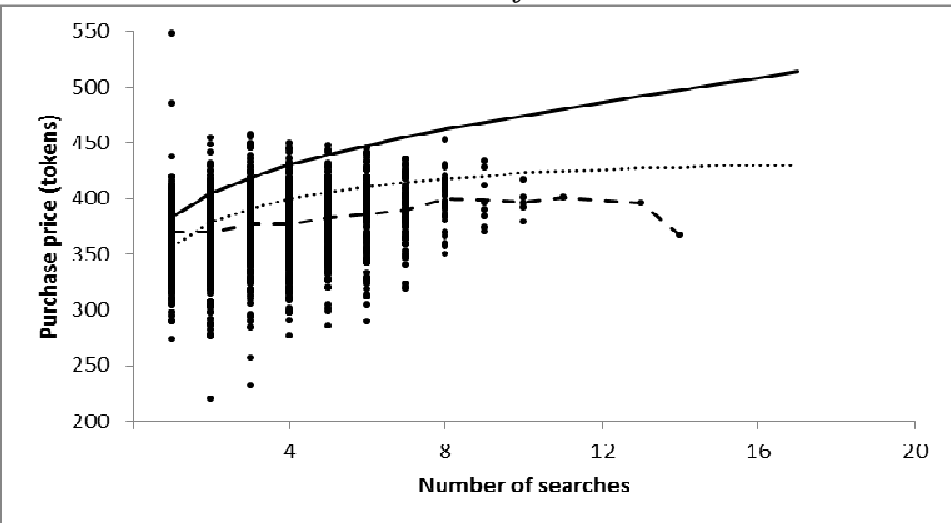

Fig. 3. Observed purchases in Experiment 2. See the caption under Fig. 1 for notations. 
Table 3. Main Results from Experiment $2(N=62)$

\begin{tabular}{ccc}
\hline \multicolumn{3}{c}{ Mean number of searches } \\
\hline English interface & Thai interface & Optimal \\
\hline$\underline{2.78}(0.23)[2.72,2.83]$ & $\underline{3.20}(0.41)[3.10,3.30]$ & 2.58 \\
$p<.001$ & Mean purchase price & \\
\hline Thai interface & Optimal \\
\hline$\underline{380}(5.23)[378,381]$ & $\underline{376}(7.24)[374,378]$ & 383 \\
$p<.001$ & $p .001$ & 3 \\
\hline
\end{tabular}

Note: See the note under Table 1 for notations, except that every pair of neighboring underlined entries highlight a significant effect of culture according to within-subjects ANOVA $(p \leq .001)$ that is discussed in more detail in the Results section for this experiment.

Table 4. Main Results from the Process Measure Questionnaire in Experiment $2(N=62)$

\begin{tabular}{ccc}
\hline Question & English interface & Thai interface \\
\hline $\begin{array}{c}\text { Q1 ("Village house" }=0, \\
\text { "Seaside hotel" }=1)\end{array}$ & $.47(.50)[.34, .60]$ & $.61(.49)[.49, .74]$ \\
Q2 ("No preference" $=0$, & & \\
$\begin{array}{c}\text { "bt65 Meal" }=1 ; \\
\text { no participant replied "bt40 Meal") }\end{array}$ & $.26(.44)[.15, .37]$ & $.42(.50)[.29, .55]$ \\
Q3 (score as in questionnaire) & $2.87(0.98)[2.62,3.12]$ & $3.21(0.89)[2.98,3.44]$
\end{tabular}

Note: See Appendix C for the complete questionnaire. The values in parentheses are the SDs and those in square brackets are $95 \%$ confidence intervals.

Table 4 suggests that, with respect to every one of the three questions, participants exhibited higher susceptibility to sunk cost-induced escalation of commitment when the question was in their Eastern (compared with Western) language. This is consistent with our theorizing. 
The standardized Cronbach's alphas for the three scores are .57 in English and .70 in Thai, suggesting poor reliability for the former and acceptable reliability for the latter. As such, we do not combine each participant's scores to form a single scale, but carry out statistical analysis in consideration of the scores as related dependent variables. With this approach, we obtain further support to our observations by a within-subjects two-factor (interface language: English vs. Thai) MANOVA with the three scores as the three dependent variables. The analysis reveals a significant main effect of interface language, Wilks' Lambda $=.91, F(1,61)=6.39, p$ $=.014, \eta_{p}^{2}=.095$. We next calculate every participant's difference in scores for each question when the question was presented in English versus Thai, through which we obtain three difference scores for each participant. We then analyze the correlations of the difference scores with the participant's corresponding differences in mean number of searches and mean purchase price by interface language. We find significant (Q1, Q2) and marginally significant (Q3) positive correlations with number of searches $(\mathrm{Q} 1: r(60)=.42,95 \% \mathrm{CI}=[.19, .60], p<.001$; Q2: $r(60)=.28,95 \% \mathrm{CI}=[.030, .49], p=.027 ; \mathrm{Q} 3: r(60)=.24,95 \% \mathrm{CI}=[-.007, .46], p=.055)$, while the correlations with purchase price are non-significantly negative $(\mathrm{Q} 1: r(60)=-.17,95 \%$ $\mathrm{CI}=[-.41, .080], p=.17 ; \mathrm{Q} 2: r(60)=-.11,95 \% \mathrm{CI}=[-.35, .14], p=.39 ; \mathrm{Q} 3: r(60)=-.043,95 \%$ $\mathrm{CI}=[-.29, .21], p=.74)$. That is, all correlations are in the theorized directions, and are largely significantly so with the number of searches. Overall, we conclude that our process measures offer consistent evidence for our theorizing; that is, Eastern (versus Western) cultural priming manipulations, as implemented by interface language in our experiment, led to increased susceptibility to sunk cost-induced escalation of commitment among our bicultural participants and an ensuing over-search bias.

\section{Discussion}


Experiment 2 lends further support to our theorizing and our results from Experiment 1, but here in a within-subjects context. In addition, our process measures provide further evidence for our theorizing, namely that escalation of commitment leads to the moderating effects of cross-cultural differences on search decisions.

\section{Experiment 3}

Experiment 3 aims to provide more concrete process evidence to our theorizing. If sunk search costs, rather than other confounding factors, drive the observed moderating effects in the previous experiments, then making sunk costs non-salient to participants, as in the present experiment, should mitigate or even eliminate any such effects.

\section{Method}

Participants. Sixty-eight UK (10 females, 8 males) and Thai (38 females, 12 males) participants, who did not take part in the previously reported experiments, participated in this experiment. The participants were recruited from the same populations as those in Experiment 1 via similar procedures. The Western and Eastern participants conducted the experiment through an interface in English and Thai respectively. The experiment had received ethical approval from the same authorities as in the pilot experiment. The participants were made aware that they would be participating, with payment, in a decision making study that would last approximately one hour.

Design and procedure. The experiment consisted of two conditions, each corresponding to a culture (UK vs. Thai). The procedures were identical to those for Experiment 1 except that sunk cost information was made non-salient at the points of decision. We made sunk costs nonsalient through removing feedback related to the total incurred search cost at all points of decision during search (see the right panel of Fig. 4); by contrast, in the pilot experiment as well 
as Experiments 1 and 2, this feedback was always provided to the participant at all points of decision (see the left panel of Fig. 4). Standard economic theorizing prescribes that the DM should only consider the tradeoff between search cost to be incurred and the expected gain from further search. The two versions of the decisions screen in Fig. 4 do not differ in their provision of such information. As a result, the benchmark optimal strategy and its predictions remain the same regardless of our sunk cost salience manipulation.

Average participant payment was $£ 6.2$ in the UK sessions and 78.5 Thai baht in the Thai sessions.

\section{Results}

The panels in Fig. 5 present the observed purchases in each condition with similar notations as in Fig. 1. The patterns of purchase observations are largely the same between the Western and Eastern participants in the present experiment, when sunk costs were non-salient (note that there are only two observations of purchases with more than nine searches among the Eastern participants in this experiment; thus the rather low purchase prices in the Thai panel corresponding to these outliers are not necessarily representative of population behavior). This observation is consistent with our premise that sunk cost effects are a key driver of the crosscultural differences in biases in search decisions. It is also consistent with Table 5, which lists the main results from the experiment and indicates whether an observed mean in a condition is significantly different from the benchmark expected value.

We combine the data from this experiment with the data from Experiment 1 pertaining only to Western participants and bicultural participants who conducted the experiment in their Eastern language (recall that the Thai participants in both experiments were from the same 


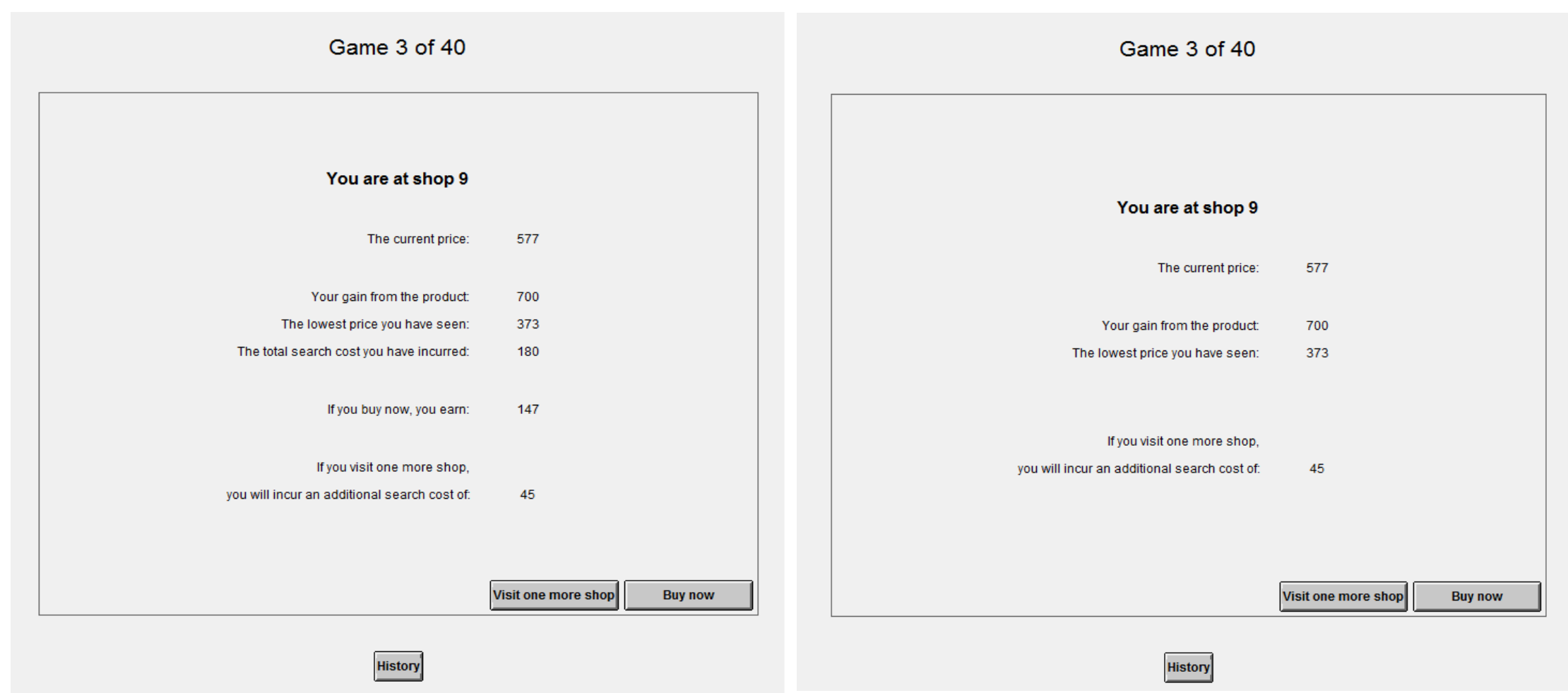

Fig. 4. Sample decision screens: sunk costs salient (Experiments 1 and 2, left) and sunk costs non-salient (Experiment 3, right). 

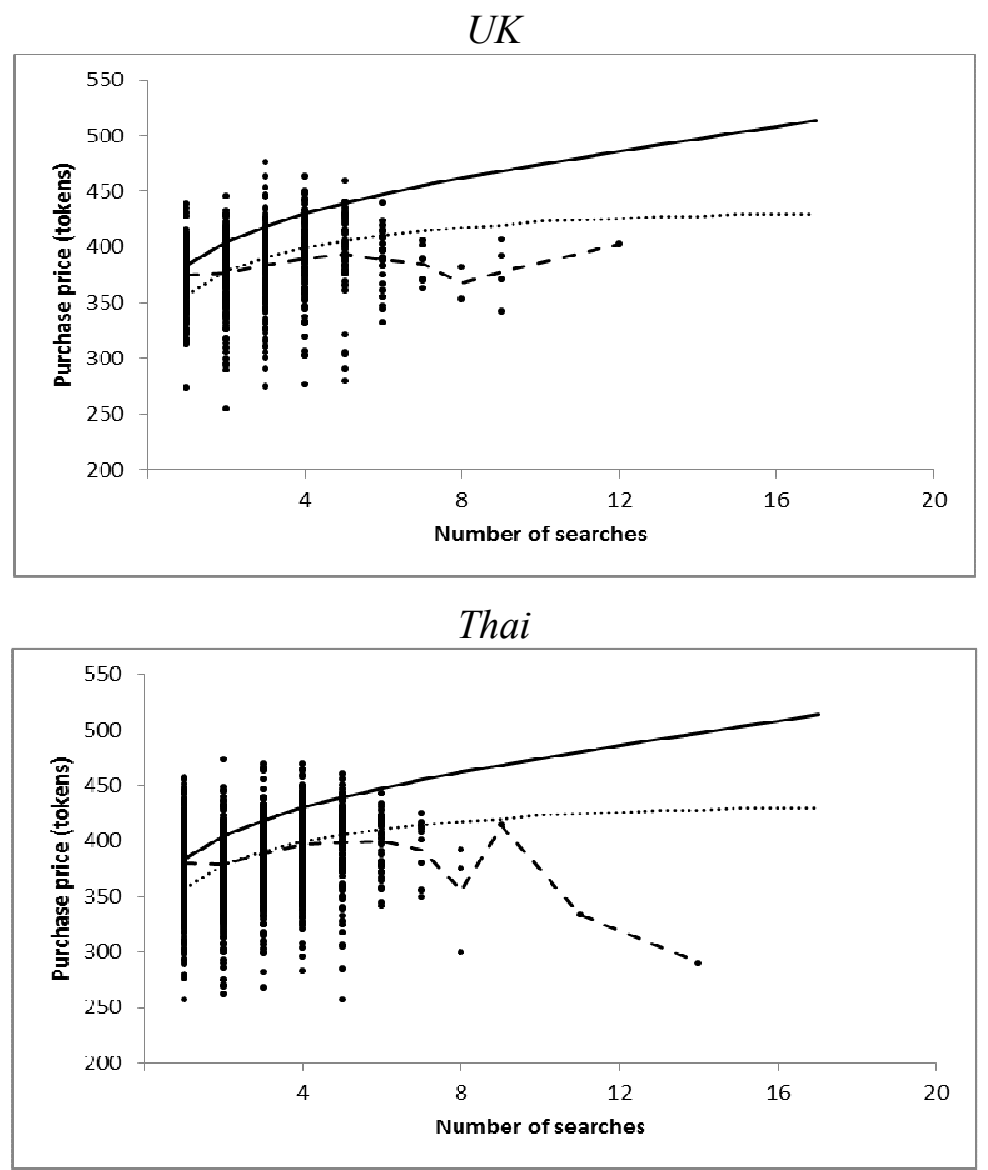

Fig. 5. Observed purchases in Experiment 3. See the caption under Fig. 1 for notations.

Table 5. Main Results from Experiment 3

\begin{tabular}{|c|c|c|}
\hline \multicolumn{3}{|c|}{ Mean number of searches } \\
\hline UK & Thai & Optimal \\
\hline $\begin{array}{c}2.73(0.51)[2.47,2.98] \\
p=.242 ; N=18\end{array}$ & $\begin{array}{c}2.52(0.43)[2.40,2.65] \\
p>.250 ; N=50\end{array}$ & 2.58 \\
\hline \multicolumn{3}{|c|}{ Mean purchase price } \\
\hline UK & Thai & Optimal \\
\hline $\begin{array}{c}\underline{381}(8.47)[377,386] \\
p>.250\end{array}$ & $\begin{array}{c}\underline{386}(6.65)[384,388] \\
p=.007\end{array}$ & 383 \\
\hline
\end{tabular}

Note: See the note under Table 1 for notations, except that the underlined entries highlight a significant effect of culture according to $t$-test $(p=.037)$ that is discussed in more detail in the Results section for this experiment. 
bicultural population). A 2 (culture: UK vs. Thai) $\times 2$ (sunk cost: salient vs. non-salient) between-subjects ANOVA for number of searches reveals a main effect of sunk cost salience $\left(F(1,149)=10.59, p=.001, \eta_{p}^{2}=.066\right)$ and an interaction effect $(F(1,149)=13.16, p<.001$, $\left.\eta_{p}^{2}=.081\right)$, while the main effect of culture is not significant $(F(1,149)=2.71, p=.102$ $\left.\eta_{p}^{2}=.018\right)$. As reported earlier for Experiment 1, the number of searches differed significantly across cultures under salient sunk costs. However, additional $t$-test shows that this simple effect went away under non-salient sunk costs $(t(66)=1.63, p=.107$, Cohen's $d=0.40)$. That is, Eastern participants in a salient sunk cost condition searched significantly more than all other conditions, in support of our theory.

A similar ANOVA for purchase price reveals only a significant interaction effect $\left(F(1,149)=18.20, p<.001, \eta_{p}^{2}=.109\right)$ but non-significant effects otherwise (main effect of sunk cost salience: $F(1,149)=2.00, p=.159, \eta_{p}^{2}=.013$; main effect of culture: $F(1,149)=1.25, p>$ $.250, \eta_{p}^{2}=.008$.) Pairwise comparisons reveal that the purchase price of the Eastern participants was significantly higher than that of the Western participants when sunk costs were non-salient $(t(66)=2.11, p=.037$, Cohen's $d=0.52)$. That is, surprisingly, making sunk costs non-salient in effect reversed the general differences in purchase prices across cultures in this research.

\section{Discussion}

Experiment 3 lends further process evidence to our theorizing by showing that, once sunk costs became non-salient, the previously observed cross-cultural effects on search decisions were largely mitigated.

\section{General Discussion}


Our research brings insights from cross-cultural studies to research on search decisions, with substantive consequences supported by process evidence involving sunk cost effects. In the pilot experiment, both Western and Eastern participants - from distinctly individualist and collectivist cultures - under-searched when search cost was low; but only the Eastern participants over-searched when search cost was high. Experiments 1 and 2 show that a change in interface language conceived as cultural priming could change bicultural participants' biases in search decisions in hypothesized directions. The process measures in Experiment 2 lend support to the theorized role of sunk cost-induced escalation of commitment in the phenomena we study. Experiment 3 provides more concrete process evidence by showing that a simple manipulation that made sunk costs non-salient could largely mitigate cross-cultural differences in search decisions.

Both our theory and experiments highlight that search decisions are not impacted straightforwardly by culture, but by an interaction of cross-cultural differences (along the individualism-collectivism dimension) and sunk search costs. Thus culture had an impact on search only in the high search cost condition in the pilot experiment, while in Experiment 3, its impact was mitigated once search costs became non-salient. This implies that our results cannot be fully explained by cross-cultural differences that may lead to only a main effect of culture on search. These include, for example, a direct (i.e., not via sunk cost effects) application of the finding that Westerners (Easterners) tended to be risk averse (risk seeking) in financial decisions (Weber \& Hsee, 1998; Hsee \& Weber, 1999); or the finding that Easterners tended to exhibit less delay discounting than Westerners (Kim, Sung, \& McClure, 2012).

We have not detected an overall impact of within-session learning in our experiments (see Appendix D), suggesting that learning or related factors cannot account for our main 
findings either. The heuristic explanations explored in previous search experiments (e.g., Bearden \& Rapoport, 2005) are also of limited use here. Those explanations were aimed at under-search rather than over-search biases, while we need to account for over-search at high search costs among collectivist participants. For example, satisficing heuristics, by which the decision maker compromises with outcomes that are "sufficiently" good, might be expected to lead to under-search only. Nevertheless, experimentation that investigates cross-cultural differences in search heuristics might provide an avenue for future research. It would also be worthwhile to test relevant hypotheses in our theorizing that are supported by previous literature but not addressed in our experiments. Finally, our experimental task, with its simple design, avails itself of further worthwhile development in computational decision making modelling. For example, we can simulate the search decisions of decision makers with various degrees of sunk cost sensitivity, and then compare with data from corresponding experimentation. 


\section{Author Contributions}

Both authors developed the study concept and design, and drafted the manuscript. Data

collection was performed by J. A. Pattaratanakun. Both authors approved the final version of the manuscript for submission.

\section{Declaration of Conflicting Interests}

The authors declared that they had no conflicts of interest with respect to their authorship or the publication of this article. 


\section{References}

Arkes, H. R., \& Blumer, C. (1985). The psychology of sunk cost. Organizational Behavior and Human Decision Processes, 35, 124-140.

Beales, H., Mazis, M. B., Salop, S. C., \& Staelin, R. (1981). Consumer search and public policy. Journal of Consumer Research, 8, 11-22.

Bearden, J. N., \& Rapoport, A. (2005). Operations research in experimental psychology. In H. J. Greenberg \& J. C. Smith (Eds), Tutorials in operations research: Emerging theory, methods, and applications (2-24). New Orleans, LA: INFORMS.

DeGroot, M. H. (1970). Optimal statistical decisions. New York: McGraw-Hill.

Dickson, P. R., \& Sawyer, A. G. (1990). The price knowledge and search of supermarket shoppers. Journal of Marketing, 54, 42-53.

Fischbacher, U. (2007). z-Tree: Zurich toolbox for ready-made economic experiments. Experimental Economics, 10, 171-178.

Gardner, W. L., Gabriel, S., \& Lee, A. Y. (1999). "I" value freedom, but "we" value relationships: Self-construal priming mirrors cultural differences in judgment. Psychological Science, 10, 321-326.

Hoffman, C., Lau, I., \& Johnson, D. R. (1986). The linguistic relativity of person cognition: An English-Chinese comparison. Journal of Personality and Social Psychology, 51, 10971105.

Hofstede, G. (1980). Culture's Consequences: International Differences in Work Related Values. London: Sage Publications.

Hofstede Centre (2014). Country comparison. Retrieved in March 2014 from http://geerthofstede.com/countries.html 
Hong, Y., Morris, M. W., Chiu, C. Y., \& Benet-Martinez, V. (2000). Multicultural minds: A dynamic constructivist approach to culture and cognition. American Psychologist, 55, 709720.

Hsee, C. K., \& Weber, E. U. (1999). Cross-national differences in risk preference and lay predictions. Journal of Behavioral Decision Making, 12, 165-179.

International Monetary Fund (2013). World economic outlook database, October 2013. Retrieved in March 2014 from http://www.imf.org/external/pubs/ft/weo/2013/02/weodata/index.aspx

Keil, M., Tan, B. C. Y., Wei, K., Saarinen, T., Tuunainen, V., \& Wassenaar, A. (2000). A crosscultural study on escalation of commitment behavior in software projects. MIS Quarterly, 24, 295-325.

Kemmelmeier, M., \& Cheng, B. Y. (2004). Language and self-construal priming: A replication and extension in a Hong Kong sample. Journal of Cross-Cultural Psychology, 35, 705-712.

Kim, B., Sung, Y. S., \& McClure, S. M. (2012). The neural basis of cultural differences in delay discounting. Philosophical Transactions of the Royal Society B, 367, 650-656.

Kogut, C. A. (1990). Consumer search behavior and sunk costs. Journal of Economic Behavior and Organization, 14, 381-392.

Luna, D., Ringberg, T., \& Peracchio, L. A. (2008). One individual, two identities: Frame switching among biculturals. Journal of Consumer Research, 35, 279-293.

Moorthy, S., Ratchford, B. T., \& Talukdar, D. (1997). Consumer information search revisited: Theory and empirical analysis. Journal of Consumer Research, 23, 263-277.

Philips, O. R., Battalio, R. C., \& Kogut, C. A. (1991). Sunk and opportunity costs in valuation and bidding. Southern Economic Journal, 58, 112-128. 
Rapoport, A., \& Tversky, A. (1970). Choice behavior in an optional stopping task. Organizational Behavior and Human Performance, 5, 105-120.

Ross, M., Xun, E. W. Q., \& Wilson, A. E. (2002). Language and the bicultural self. Personality and Social Psychology Bulletin, 28, 1040-1050.

Seale, D., \& Rapoport, A. (1997). Sequential decision making with relative ranks: An experimental investigation of the "secretary problem." Organizational Behavior and Human Decision Processes, 67, 258-270.

Sui, J. \& Han, S. (2007). Self-construal priming modulates neural substrates of self-awareness. Psychological Science, 18, 861-866.

Thaler, R. H. (1980).Toward a positive theory of consumer choice. Journal of Economic Behavior and Organization, 1, 39-60.

Triandis, H. C. (1995). Individualism and Collectivism. Boulder, CO: Westview Press.

Urbany, J. E., Dickson, P. R., \& Kalapurakal, R. (1996). Price search in the retail grocery market. Journal of Marketing, 60, 91-104.

Weber, E. U., \& Hsee, C. K. (1998).Cross-cultural differences in risk perception, but crosscultural similarities in attitudes towards perceived risk. Management Science, 44, 12051217.

Weitzman, M. L. (1979). Optimal search for the best alternative. Econometrica, 47, 641-654.

Zwick, R., \& Rapoport, A., Lo, A. K. C., \& Muthukrishnan, A. V. (2003). Consumer sequential search: Not enough or too much? Marketing Science, 22, 503-519. 


\section{Culture Moderates Biases in Search Decisions}

Supplemental Material

\section{Appendix A: Optimal Search Strategies in the Experiments}

The search tasks in all our experiments share the following feature: if the decision maker (DM) stops after obtaining exactly $t$ price quotes, his/her payoff can be expressed as:

$$
\pi_{t}\left(p_{1}, p_{2}, p_{3}, \ldots p_{t}\right)=u-\min \left\{p_{1}, p_{2}, p_{3}, \ldots p_{t}\right\}-\sum_{i=0}^{t-1} c_{i}
$$

where $p_{i}(i=1,2,3, \ldots t)$ denotes the $i$-th price quote obtained, $c_{0}=0, c_{i}(i>0)$ denotes the cost to obtain one more price quote when the DM has already obtained $i$ price quotes, and $u$ (= 700 tokens in all experiments) is the value of the purchased product to the DM.

\section{The pilot experiment}

The search task in the pilot experiment has constant search costs, i.e., $c_{i}=c=$ constant for all $i>0$. The optimal search strategy - defined as the strategy that maximizes expected payoff - is well known (see e.g., DeGroot, 1970). Suppose the price quotes are distributed according to the cumulative probability function $F(\cdot)$. Then the decision maker should accept the current minimum price quote and stop the search, if and only if the current minimum price quote becomes not higher than a threshold price $p^{*}$, where $p^{*}$ is determined by the following implicit equation:

$$
c-\int_{-\infty}^{p^{*}}\left(p^{*}-p\right) d F(p)=0
$$

In the pilot experiment, $c=5$ tokens or 15 tokens while $F(\cdot)$ is a normal distribution with mean $=430$ tokens and standard deviation $=50$ tokens. The corresponding threshold prices can be obtained numerically using the above equation. In practice, the threshold prices discussed in the main text are the highest integer prices at which the left hand side of the above equation remains 
positive. The expected number of searches, the expected purchase price, as well as the expected payoff under the optimal strategy can be computed numerically using the threshold prices; the results are listed in Table 1.

\section{Experiments 1 to 3}

The search task in Experiments 1 to 3 has increasing search costs in the form $c_{i}=k \cdot i$ where $k$ is a positive constant. The optimal strategy should involve an increasing sequence of threshold prices $p_{1}{ }^{*}, p_{2}{ }^{*}, \ldots p_{i}{ }^{*} \ldots$, such that a decision maker should stop the search immediately after $i$ searches if and only if the current minimum price quote becomes not higher than $p_{i}{ }^{*}$ (see e.g., Kohn, M. G., \& Shavell, S. (1974). The theory of search. Journal of Economic Theory, 9, 93-123). In particular, if the current minimum price quote is exactly $p_{i}{ }^{*}$, the decision maker should be indifferent between continuing search and stopping. But since the threshold prices are an increasing sequence, even if the decision maker makes one more search, he/she must stop after the next search, since the minimum price quote at the point immediately after the next search cannot be higher than $p_{i}{ }^{*}$, which is itself lower than $p_{i+1} *$. In fact, there is a probability $F\left(p_{i}{ }^{*}\right)$ that the decision maker would obtain a lower price quote than $p_{i}{ }^{*}$ in the next search, in which case the conditional expected value of that price quote would be $\int_{-\infty}^{p_{i}{ }^{*}} p d F(p) / F\left(p_{i}{ }^{*}\right)$. There is at the same time a probability $1-F\left(p_{i}{ }^{*}\right)$ that the minimum price quote immediately after the next search remains $p_{i}{ }^{*}$. To sum up, we must have:

$$
u-p_{i}{ }^{*}=-k \cdot i+F\left(p_{i}{ }^{*}\right)\left[u-\frac{\int_{-\infty}^{p_{i}{ }^{*}} p d F(p)}{F\left(p_{i}{ }^{*}\right)}\right]+\left[1-F\left(p_{i}{ }^{*}\right)\right]\left(u-p_{i}{ }^{*}\right)
$$

which becomes, after simplification: 


$$
k \cdot i-\int_{-\infty}^{p_{i}^{*}}\left(p_{i}^{*}-p\right) d F(p)=0 .
$$

To check for consistency, differentiation of the right hand side as a function in $p_{i} *$ yields:

$$
\frac{\partial}{\partial p_{i}^{*}} \int_{-\infty}^{p_{i}^{*}}\left(p_{i}^{*}-p\right) d F(p)=0+\int_{-\infty}^{p_{i}^{*}} d F(p)=F\left(p_{i}^{*}\right)
$$

which must be positive for values of $p_{i} *$ that solve the threshold price equation above. Thus the right hand side must be a strictly increasing function in $p_{i} *$ in general. Since $k \cdot i$ increases with $i$, the threshold prices must be strictly increasing in $i$ as well. The threshold prices for our setup in Experiments 1 to 3 are obtained numerically using (A1) and are as discussed in the main text. In practice, the threshold prices in Table A1 are the highest integer prices at which the left hand side of (A1) remains positive, and as such are the thresholds for acceptance (i.e., stopping search). The expected number of searches, the expected purchase price, as well as the expected payoff under the optimal strategy can be computed numerically using the threshold prices; the results are listed in Tables 2, 3, and 5. 
Table A1. Threshold Prices Under the Optimal Strategy in Experiments 1 to 3

\begin{tabular}{ccc}
\hline $\begin{array}{c}\text { Number of searches } \\
\text { already carried out }\end{array}$ & $\begin{array}{c}\text { Cost in tokens for } \\
\text { one additional search }\end{array}$ & $\begin{array}{c}\text { Threshold price in tokens } \\
\text { for stopping }\end{array}$ \\
\hline 1 & 5 & 384 \\
2 & 10 & 405 \\
3 & 15 & 419 \\
4 & 20 & 430 \\
5 & 25 & 439 \\
6 & 30 & 447 \\
7 & 35 & 455 \\
8 & 40 & 462 \\
9 & 45 & 468 \\
10 & 50 & 474 \\
11 & 55 & 480 \\
12 & 60 & 486 \\
13 & 65 & 492 \\
14 & 70 & 497 \\
15 & 75 & 503 \\
16 & 80 & 508 \\
17 & 85 & 514 \\
\hline & &
\end{tabular}

Note: The threshold prices are defined such that an expected payoff-maximizing DM should accept the current minimum price quote (i.e., stop search) upon having made $i$ searches, if that price quote is lower than or equal to $p_{i}{ }^{*}$. The threshold prices corresponding to $c_{i}=5$ tokens and 15 tokens are also the threshold prices under the optimal strategy in the low and high search cost conditions in the pilot experiment, respectively. 


\section{Appendix B: Sample Experimental Instructions}

(UK/Low Search Cost Condition, the pilot experiment)

\section{SEARCH GAME}
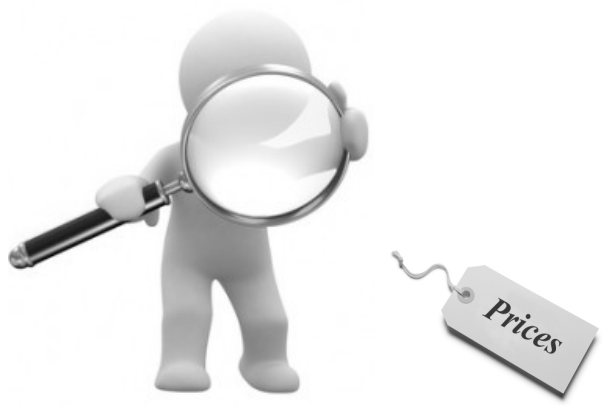

\section{INSTRUCTIONS}

In this study you will make many decisions. Your payment at the end of the study will depend on your decisions and luck.

In case you have any questions, please raise your hand and the study coordinator will come to answer them.

Please now switch off your mobile phone and all other communicating devices. For the duration of the study, you are not allowed to communicate with other participants.

\section{Description of the Game}

During this study, you will play the same game 45 times.

In each game, you visit one or more shops to buy one product item. When you visit a shop, you will see the price of the product at that shop. After every visit, you can decide whether to visit one more shop or to buy the product. Once you decide to buy the product, the game will end immediately.

To buy the product, you need to pay the lowest price among the shops that you have visited. On the other hand, you gain 700 tokens (the experimental currency used in this study that will be later converted to real money) from the product itself. 
In addition, there is a search cost: after visiting the first shop, you have to pay 5 tokens for each additional shop that you visit.

Therefore your earnings in the game is:

700 tokens - The lowest price among the shops that you have visited

- The total search cost of your visits

\section{Example}

You have just visited shop 4. The prices at the shops you have visited, and the costs of each visit, are as follow:

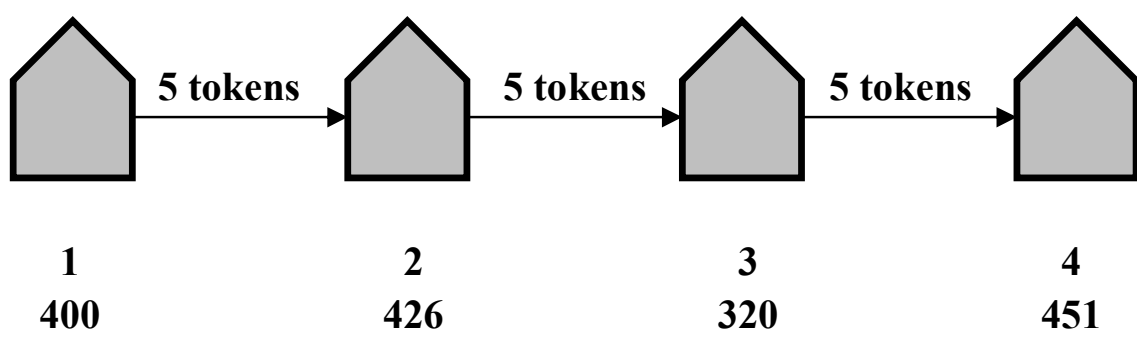

$\begin{array}{lcccc}\text { Shop } & 1 & 2 & 3 & 4 \\ \text { Price } & 400 & 426 & 320 & 451\end{array}$

(tokens)

That is, it costs you 5 tokens to visit shop 2, another 5 tokens to visit shop 3, and another 5 tokens to visit shop 4. The total search cost of your visits is therefore 5+5+5=15 tokens.

You now decide to buy. The price you pay is then 320 tokens, which is the lowest price among the four shops you have visited. Your earnings in this game are therefore:

700 tokens -320 tokens -15 tokens $=\underline{365 \text { tokens }}$

\section{How the prices are distributed among the shops}

The prices at the shops are independent from each other. However, they all follow a normal ("bell-shaped") distribution with average of 430 tokens and standard deviation of 50 tokens. In the Appendix you can find a table and a graph charting the approximate chances that the price at a shop is in various price ranges. 


\section{Procedures}

You will enter all your decisions via the computer terminal in front of you.

During each game, you will see on the computer a decision screen such as Figure 1 (all the figures are on p.5 and p.6). The decision screen lists the following from top to bottom:

1. The number of the game you are playing

2. The number of the shop you are visiting

3. The price at the shop you are visiting ("The current price")

4. The lowest price you have seen from your visits

5. The total search cost that you have incurred from your visits

6. Your earnings if you buy now

7. The additional search cost that you will incur if you visit one more shop

8. The buttons that correspond to the decision you can make i.e. "Visit one more shop" or "Buy now"

9. A "History" button which you can click to see a history screen like Figure 2, which lists the prices at all the shops you have visited. When you are at a history screen, you can click the "Go back" button at the bottom of the screen to go back to the decision screen.

To make a decision, click the button labeled with your choice i.e. either "Visit one more shop" or "Buy now".

After you click the "Buy now" button, the game ends and you will see on the computer a summary screen for the game, like Figure 3 .

First, you will play 5 practice games that will not count towards your cash payment. Then you will play 40 games for payment; of these games, the program will pick 3 games at random and pay you your average earnings from those 3 games at a rate of:

$$
1 \text { token }=£ 0.01 .
$$

In addition, you will be paid a show-up fee of $£ 3$.

If you don't have any questions, please complete the quiz in the next page. We shall come and check your answers shortly. 
Figure 1.The decision screen

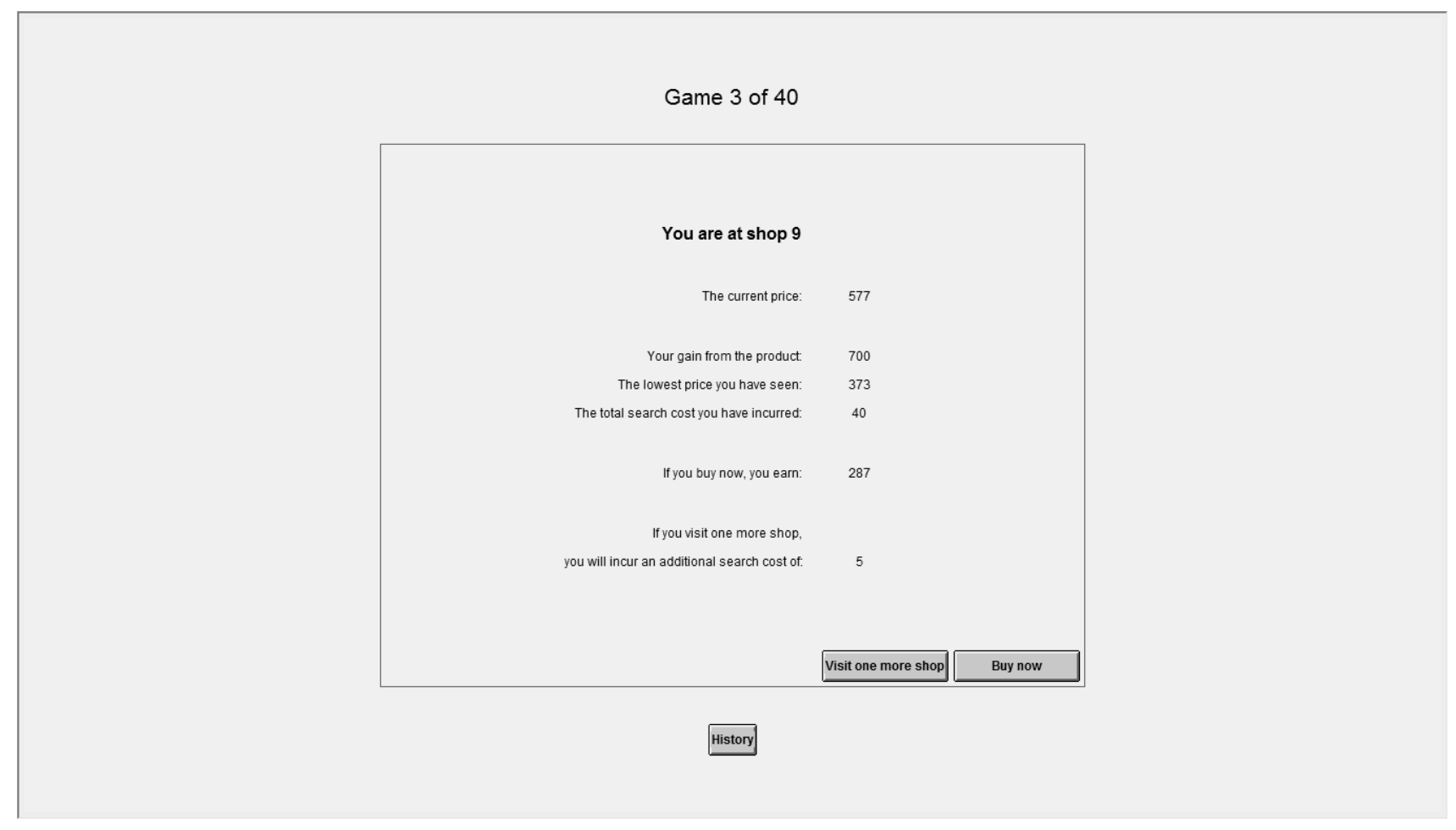

Figure 2.The history screen

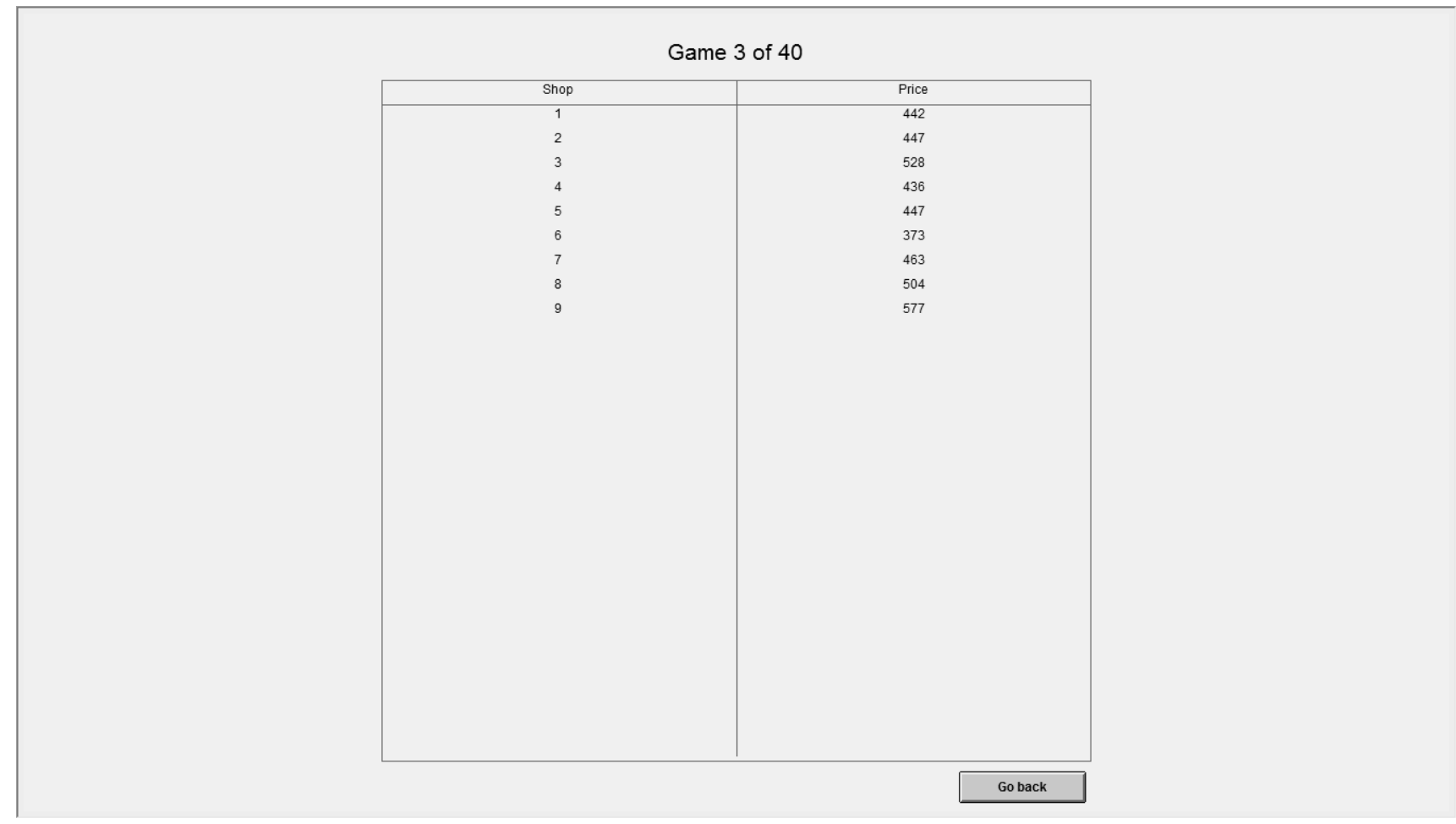


Figure 3 . The summary screen

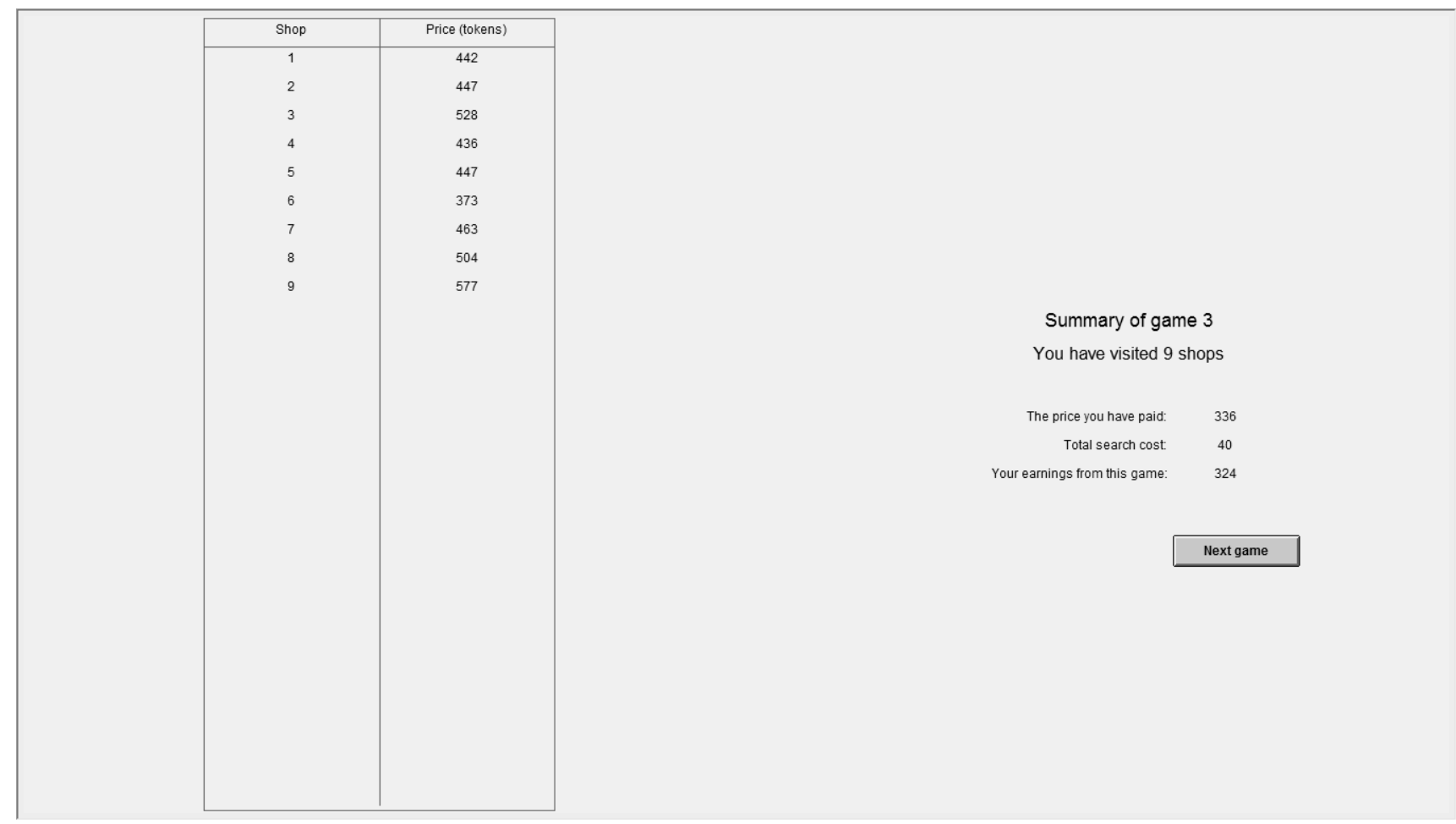




\section{QUIZ}

Please fill in the blanks below.

1. On average, the price at a shop is tokens.

2. There is a $\%$ chance that the price at a shop is between 370 and 380 tokens.

3. There is a $\%$ chance that the price at a shop is between 460 and 470 tokens.

4. __ games will be chosen randomly from games to calculate your cash payment.

5. Suppose you have just visited shop 5 and you find that:

The price at shop 1 is 343 tokens;

The price at shop 2 is 242 tokens;

The price at shop 3 is 410 tokens;

The price at shop 4 is 443 tokens;

The price at shop 5 is 489 tokens.

If you buy now, the price you pay is tokens. The total search cost of your visits is tokens. Yours earnings are tokens.

If you wish to participate in this study, please sign the consent form, and then wait patiently until all other players are ready to start. 


\section{APPENDIX}

The chances that the price at a shop is in various price ranges

\section{In Table Format:}

\begin{tabular}{|c|c|}
\hline Price range in tokens & Percentage chance that the price at a shop is in this range \\
\hline$<330$ & $2.3 \%$ \\
\hline $330-340$ & $1.3 \%$ \\
\hline $340-350$ & $1.9 \%$ \\
\hline $350-360$ & $2.6 \%$ \\
\hline $360-370$ & $3.4 \%$ \\
\hline $370-380$ & $4.4 \%$ \\
\hline $380-390$ & $5.3 \%$ \\
\hline $390-400$ & $6.2 \%$ \\
\hline $400-410$ & $7.0 \%$ \\
\hline $410-420$ & $7.6 \%$ \\
\hline $420-430$ & $7.9 \%$ \\
\hline $430-440$ & $7.9 \%$ \\
\hline $440-450$ & $7.6 \%$ \\
\hline $450-460$ & $7.0 \%$ \\
\hline $460-470$ & $6.2 \%$ \\
\hline $470-480$ & $5.3 \%$ \\
\hline $480-490$ & $4.4 \%$ \\
\hline $490-500$ & $3.4 \%$ \\
\hline $500-510$ & $2.6 \%$ \\
\hline $510-520$ & $1.9 \%$ \\
\hline $520-530$ & $1.3 \%$ \\
\hline$>530$ & $2.3 \%$ \\
\hline
\end{tabular}




\section{In Graph Format:}

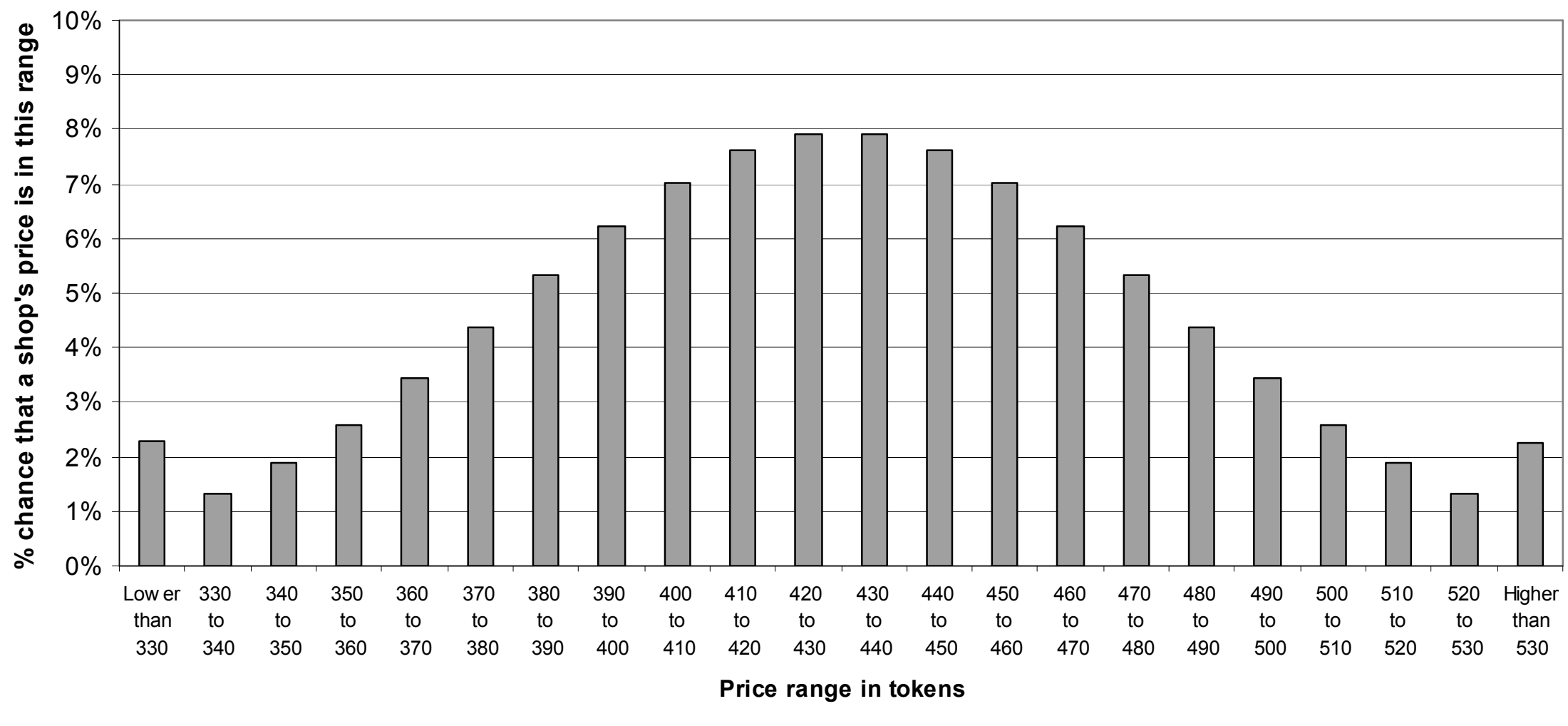




\section{Appendix C: Process Measure Questionnaire for Experiment 2}

\section{For each of the following questions, please tick the box next to your answer:}

Q1: Imagine that a month ago, you made a nonrefundable deposit of bt1,300 on a weekend package at a seaside hotel. Since the reservation was made, however, you have been invited to spend the same weekend at a friend's village house in the countryside. You would prefer to spend the weekend at the village house but if you don't go to the seaside hotel, the bt1,300 deposit will be lost. Where would you spend the weekend?

$\square$ Village house $\quad \square$ Seaside hotel

Q2: Imagine that on your way home you buy a microwave meal on sale for bt40 at the local grocery store. A few hours later you decide it is time for dinner, so you get ready to put the food in the oven. Then you get an idea. You call up your friend to ask if he would like to come over for a quick dinner and then watch a good movie on TV. Your friend says "Sure." So you go out to buy a second microwave meal. However, all the on-sale microwave meals are gone. You therefore have to spend bt65 (the regular price) for the microwave meal identical to the one you just bought for bt40. You go home and put both dinners in the oven. When the two dinners are fully cooked, you get a phone call. Your friend is ill and cannot come. You are not hungry enough to eat both dinners. You cannot freeze one. You must eat one and discard the other. Which one do you eat?

$\square$ bt40 Meal $\quad \square$ bt65 Meal $\quad \square$ No preference

Q3: You join a tennis club and pay a bt3,900 yearly membership fee. After two weeks of playing you develop a tennis elbow. On a scale of 1 to 5 where 5 represents "very likely" and 1 represents "very unlikely," how likely would you continue to play (in pain)?
$\square 5$
$\square 4$
$\square 3$
$\square 2$ 


\section{Appendix D: Learning Analysis}

For every experimental condition in our study, we test whether there was significant within-session learning among participants. To proceed, we divide the 40 games for payment in the session into two blocks, so that Block 1 includes Game 1 to 20 and Block 2 includes Game 21 to 40. We then conduct, for each condition, a two-factor (Block 1 versus Block 2) withinsubject MANOVA with the number of searches and the purchase price as the dependent variables and participant as the unit of observation. The conditions include four from the pilot experiment, three from Experiment 1, four from Experiment 2 (defined by the $2 \times 2$ design discussed at the beginning of its Results section), and two from Experiment 3.

We focus on testing for main effect of block; the results are summarized in Table A2. The tests do not yield a significant effect of block in almost every condition, with the single exception of one condition in Experiment 2, namely the Thai interface session under the Thai then English order. Simple effect tests for effect of block on the two dependent variables in that condition do not yield a significant effect for number of searches $(F(1,31)=0.34, p>.250$, $\left.\eta_{p}^{2}=.011\right)$ but a significant effect for purchase price $\left(F(1,31)=5.88, p=.021, \eta_{p}^{2}=.159\right)$. Further examination shows that, even though there was some ostensible learning effect in this particular case, it only amounted to a decrease of 0.070 in the average number of searches $(2.09 \%$ of the average number of searches in Block 1), and a decrease of 5.01 tokens in the average purchase price $(1.32 \%$ of the average purchase price in Block 1$)$, across blocks.

We thus conclude that we have not detected an overall impact of within-session learning effects in our experiments. 
Table A2. Test Results for Effect of Block in the Experimental Conditions

\begin{tabular}{|c|c|c|c|c|}
\hline Condition & Wilks' Lambda & $F$ & $p$ & $\eta_{p}^{2}$ \\
\hline \multicolumn{5}{|c|}{ Pilot experiment } \\
\hline UK/Low search cost & .933 & $F(1,21)=1.52$ & .232 & .067 \\
\hline UK/High search cost & .996 & $F(1,22)=0.10$ & $>.250$ & .004 \\
\hline Thai/Low search cost & .913 & $F(1,22)=2.10$ & .162 & .087 \\
\hline Thai/High search cost & .997 & $F(1,39)=0.10$ & $>.250$ & .003 \\
\hline \multicolumn{5}{|c|}{ Experiment 1} \\
\hline UK & 1.00 & $F(1,35)=0.00$ & $>.250$ & $<.001$ \\
\hline Thai (English interface) & 1.00 & $F(1,34)=0.00$ & $>.250$ & $<.001$ \\
\hline Thai (Thai interface) & 1.00 & $F(1,48)=0.03$ & $>.250$ & $<.001$ \\
\hline \multicolumn{5}{|c|}{ Experiment 2} \\
\hline English then Thai/English interface & 1.00 & $F(1,29)=0.00$ & $>.250$ & $<.001$ \\
\hline English then Thai/Thai interface & .986 & $F(1,29)=0.41$ & $>.250$ & .014 \\
\hline Thai then English/English interface & .997 & $F(1,31)=0.08$ & $>.250$ & .003 \\
\hline Thai then English/Thai interface & .833 & $F(1,31)=6.22$ & .018 & .167 \\
\hline \multicolumn{5}{|c|}{ Experiment 3} \\
\hline UK & 1.00 & $F(1,17)=0.02$ & $>.250$ & .001 \\
\hline Thai & 1.00 & $F(1,49)=0.05$ & $>.250$ & .001 \\
\hline
\end{tabular}

